\title{
Observation of Magnetic Anisotropy in GaN:Cr Single Crystals
}

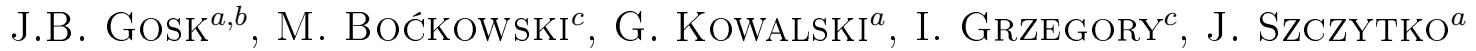 \\ AND A. TWARDOWSKI ${ }^{a}$ \\ ${ }^{a}$ Institute of Experimental Physics, Faculty of Physics, University of Warsaw, Hoża 69, 00-681 Warsaw, Poland \\ ${ }^{b}$ Faculty of Physics, Warsaw University of Technology, Koszykowa 75, 00-662 Warsaw, Poland \\ ${ }^{c}$ High Pressure Research Center, PAS, Sokołowska 29/37, 01-142 Warsaw, Poland
}

\begin{abstract}
Magnetic properties of bulk wurtzite GaN:Cr single crystals were studied with the magnetic field applied parallel and perpendicular to the crystal wurtzite $c$-axis. Structure of the crystal was examined by the X-ray diffraction method. Strong anisotropy of magnetization at low temperatures $(2-10 \mathrm{~K})$ was observed. The experimental data suggest $\mathrm{Cr}$ to be in nonspherical $d^{4}$ configuration.
\end{abstract}

PACS: 71.55.Eq, 75.30.Gw, 75.50.Pp, 75.10.Dg

\section{Introduction}

Over the last decade GaN doped with transition-metals (TM) has been extensively studied both experimentally and theoretically. Among the prominent candidates for future spintronics are GaN:Mn and GaN:Cr. However, there is still lack of sufficient experimental data on diluted magnetic semiconductors (DMS) based on Cr, especially on paramagnetic phase of GaN:Cr [1, 2]. The electron configuration of free $\mathrm{Cr}$ atom is $[\mathrm{Ar}] 3 d^{4} s^{2}$ and neutral electronic configuration of $\mathrm{Cr}$ substituting $\mathrm{Ga}$ in $\mathrm{GaN}$ is $\mathrm{Cr}^{3+}\left(d^{3}\right)$. There are reports that $\mathrm{Cr}$ substituting $\mathrm{Ga}$ ions in the $\mathrm{GaN}$ lattice forms $\mathrm{Cr}^{2+} /{ }^{3+}$ deep acceptor level about $2 \mathrm{eV}$ above $\mathrm{GaN}$ valence band [3, 4]. Since, in $n$-type GaN the Fermi level is located near the conduction band, the expected electron structure of $\mathrm{Cr}$ ion is $\mathrm{Cr}^{2+}\left(d^{4}\right)$. Such electronic configuration is non-spherical and should result in pronounced magnetic anisotropy. Similar situation was reported for GaN:Mn, co-doped with $\mathrm{Mg}$ [5], where manganese was found to form $\mathrm{Mn}^{3+}\left(d^{4}\right)$ center and strong magnetic anisotropy was observed [5]. In this communication we report on magnetic anisotropy of $\mathrm{Cr}$ in $\mathrm{GaN}$, which we assign to $\mathrm{Cr}^{2+}\left(d^{4}\right)$ centers.

\section{Sample preparation and measurement techniques}

In the present study bulk single crystals of $\mathrm{GaN}: \mathrm{Cr}$ were used, as for magnetic anisotropy investigation, it is crucial to have the access to the large high quality crystals. The bulk crystals are advantageous over those grown as epilayers on a substrate $[6,7]$. First, there is no any additional magnetic contribution to the total measured magnetization from the substrate which could render analysis of the data (especially at low TM contribution) difficult. Next, there is no strain-induced magnetic anisotropy originating from the lattice mismatch between GaN:Cr layer and the substrate. Finally, in the case of very low Cr content large volume bulk crystals allow us to achieve good experimental accuracy.

Strain-free GaN:Cr single crystals of wurtzite $(2 \mathrm{H}$, hexagonal) structure were grown at High Pressure Research Center UNIPRESS by the same equilibrium high pressure technique used before to grow GaN:Mn and GaN:Mn:Mg [5], i.e., by equilibrium high pressure technique from nitrogen solution in liquid gallium [8]. Chromium was added into liquid gallium. The growth was performed under high pressure of $\mathrm{N}_{2}(p \approx 1.5 \mathrm{GPa})$ and temperatures $T \approx 1500{ }^{\circ} \mathrm{C}$. The obtained crystals were platelets with diameter of about few millimeters with wurtzite $c$ axis perpendicular to the plate plane. The concentration of $\mathrm{Cr}$ in the studied samples was evaluated from magnetization data as before [5] and was in the range from 0.003 to $0.0075 \mathrm{~mol} . \%$.

We note that $n$-type GaN:Mn $\left(n \approx 10^{19} \mathrm{~cm}^{-3}\right)$ revealed paramagnetic (PM) Brillouin-type behaviour typical for $\mathrm{Mn}^{2+}\left(d^{5}\right)$. On the other hand, highly resistive GaN:Mn:Mg (with $\rho \approx 10^{19} \Omega$ at room temperature) showed high magnetic anisotropy well described by $\mathrm{Mn}^{3+}\left(d^{4}\right)$ model [5].

The structure and external morphology of GaN:Cr was examined with the use of X-ray Laue method and X-ray reflection topography employing Lang type camera in reflection mode with Mo $K_{\alpha_{1}}$ laboratory X-ray source. Magnetization was measured using SQUID magnetometer in the temperature range from $2 \mathrm{~K}$ to $400 \mathrm{~K}$ and 
magnetic field $(B)$ up to $7 \mathrm{~T}$. Single plates $(\approx 20 \mathrm{mg})$ or a few plates $(\approx 75 \mathrm{mg})$ sandwiched with the use of diamagnetic glue were attached to diamagnetic straw by the glue. The magnetic field was applied in perpendicular $(B \perp c)$ or parallel $(B \| c)$ orientation to the $\mathrm{GaN}$ hexagonal $c$-axis. All results were corrected for diamagnetic contribution of host GaN lattice and diamagnetic glue.

\section{Results and discussion}

Structure of $\mathrm{GaN}$ :Cr was investigated by X-ray Laue method as well as reflection topography (Fig. 1). Additionally optical micrograph was taken in transmission mode to show external morphology of the crystal (Fig. 2). The Laue images were recorded in few positions along the whole crystal to evaluate possible angular misorientation of the different parts of the crystal showing complicated external morphology clearly visible in Fig. 2. We can conclude that, within the setting accuracy of the Laue method $(0.5 \mathrm{deg})$ no misorientation was recorded. Whole bulk of the crystal shows regular hexagonal pattern. In some parts (Fig. 2) there can be clearly visible the underlying hexagonal structure with [0001] direction perpendicular to large face of the crystal (basically the plane of the optical micrograph). We have also taken 004 type, reflection topography with Mo $K_{\alpha_{1}}$ laboratory X-ray source (Fig. 1) to probe the internal volume of the sample. It shows quite uniform contrast inside the whole volume of the sample. The only black features shown as lines in Fig. 1 result from the sharp edges on the face of the sample. There is also no sign of any small angular misalignments between the different parts of the crystal. Since the reflection topography is sensitive to the angular setting within the angular range of the crystal, so-called rocking curve, we are well beyond the $0.5 \mathrm{deg}$ as found from the Laue experiment and probing the angles in the range of hundreds of seconds of arc. So we can finally conclude that the sample is fairly uniform single crystal of hexagonal GaN.

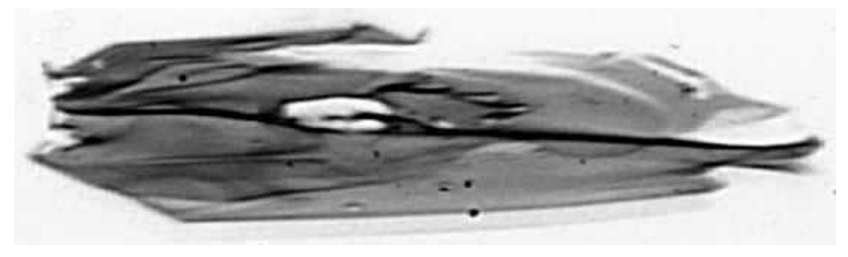

Fig. 1. An X-ray 004 reflection topography of $\mathrm{GaN}$ : $\mathrm{Cr}$ sample \#4.

Representative magnetization data are shown in Fig. 3 , where magnetization at magnetic field $B=1 \mathrm{~T}$ is shown as a function of temperature, for $B$ applied parallel and perpendicular to crystal hexagonal axis. Magnetic anisotropy is clearly visible (hexagonal axis is the hard axis), especially at low temperatures. On the other hand, at high temperatures magnetization does not decay as $1 / T$ but approaches nearly constant, positive value. This

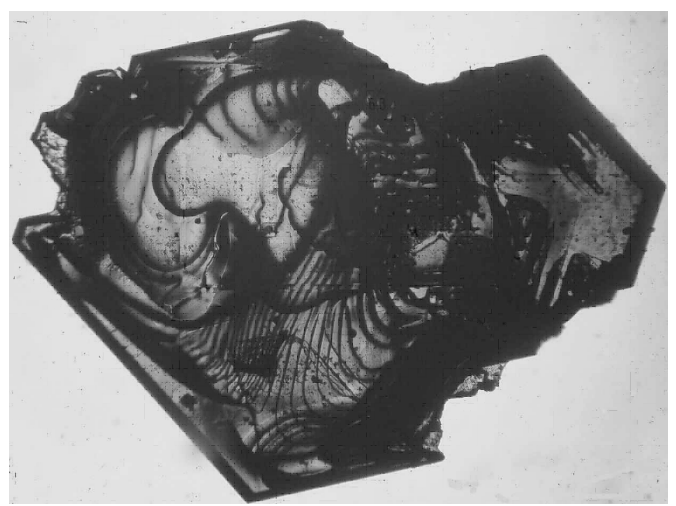

Fig. 2. Optical micrograph of the GaN:Cr sample \#4.

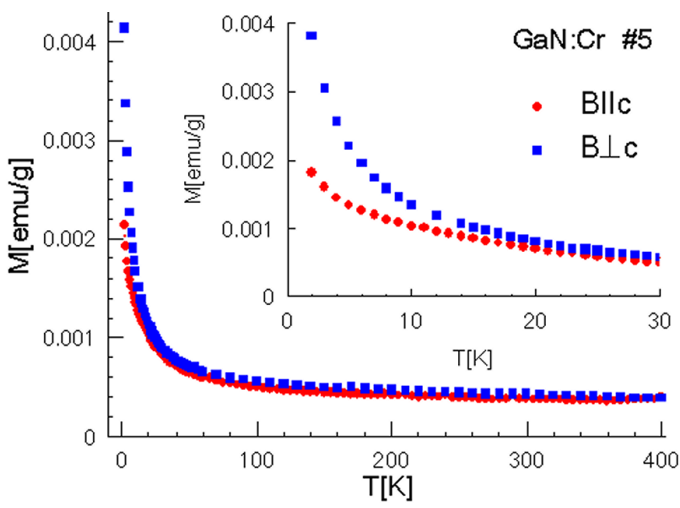

Fig. 3. Temperature dependence of magnetization at $B=1 \mathrm{~T}$ for $\mathrm{GaN}$ :Cr sample \#5 after correction for diamagnetic contribution. The inset shows the magnetization vs. temperature at low temperatures after correction for diamagnetic and FM contributions.

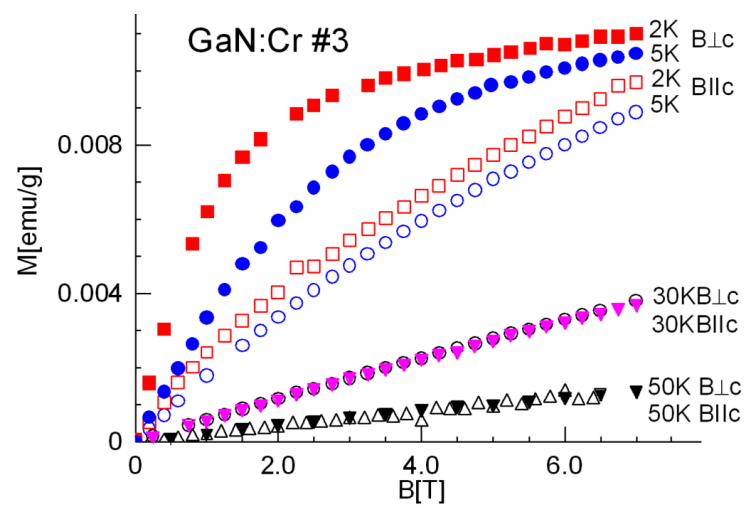

Fig. 4. Magnetization of GaN:Cr plotted as a function of the magnetic field at different temperatures after correction for FM. The closed squares, circles and triangles represent measurements at $B \perp c$ configuration while open ones represent $B \| c$ configuration. 
suggests the presence of ferromagnetic precipitations in our crystals. Such situation is commonly found in GaN-based DMS [9]. Detailed analysis of the $M(B)$ data allows a reasonable subtraction of the FM phase contribution [1]. Such correction was performed for all our data.

Figure 4 shows paramagnetic (PM) contribution to the measured magnetization, which originates from $\mathrm{Cr}$ dopant in GaN matrix. The PM contribution measured at $B \perp c$ (the close experimental points in Fig. 4) shows typical Brillouin-type behaviour: strong temperature dependence, pronounced tendency to saturation with magnetic field at the lowest temperatures, and linear field dependence at high temperatures. We note that although magnetization at temperature $T=2 \mathrm{~K}$ could be fitted by a Brillouin function (returning for sample \#3 spin $S=1.7$ and Cr concentration $x=0.000043$ ), one set of parameters (for selected field orientation) does not provide acceptable description of the data in the entire temperature and magnetic field range.

Quite different character of magnetization curves is observed when magnetic field was parallel to the GaN hexagonal $c$-axis (open experimental points in Fig. 4). For low temperatures (see curves for $T=2 \mathrm{~K}, 5 \mathrm{~K}$ ) magnetization shows much weaker tendency to saturation than in the case when magnetic field was oriented perpendicular to $c$-axis. Thus, from the data one can conclude that hard magnetization axis and easy magnetization axis are parallel and perpendicular to $c$-axis, respectively. The anisotropy decreases with increasing temperature and practically disappears above about $30 \mathrm{~K}$. This observation is in agreement with the results of temperature dependent magnetization measurements (Fig. 3).

The observed magnetic behaviour is very similar to that found for GaN:Mn:Mg [5] and CdS:Cr [10, 11], where magnetic centers were identified as $\mathrm{Mn}^{3+}\left(d^{4}\right)$ and $\mathrm{Cr}^{2+}\left(d^{4}\right)$, respectively. Basing on this similarity we suggest that the dominant electronic configuration of $\mathrm{Cr}$ in GaN is $\mathrm{Cr}^{2+}\left(d^{4}\right)$, contrary to the simple expectations of substitutional $\mathrm{Cr}^{3+}\left(d^{3}\right)$ configuration. Further support for this conclusion, provided by crystal-field model of $d^{4}$ center in GaN will be presented elsewhere.

\section{Acknowledgments}

Project carried out with the use of CePT infrastructure financed by the European Union - the European Regional Development Fund within the Operational Programme "Innovative economy" for 2007-2013.

\section{References}

[1] M. Zając, J.B. Gosk, E. Grzanka, S. Stelmakh, M. Palczewska, A. Wysmołek, K. Korona, M. Kaminska, A. Twardowski, J. Alloys Comp. 456, 324 (2008).

[2] A. Wolos, A. Kaminska, Semicond. Semimetals 82, 325 (2008).

[3] A.Y. Polyakov, N.B. Smirnov, A.V. Govorkov, N.V. Pashkova, A.A. Shlensky, S.J. Pearton, M.E. Overberg, C.R. Abernathy, J.M. Zavada, R.G. Wilson, J. Appl. Phys. 93, 5388 (2003).

[4] U. Gertsmann, A.T. Blumenau, H. Overhof, Phys. Rev. B 63, 075204 (2001).

[5] J.B. Gosk, M. Zajac, A. Wolos, M. Kaminska, A. Twardowski, I. Grzegory, M. Bockowski, S. Porowski, Phys. Rev. B 71, 094432 (2005).

[6] J.J. Kim, H. Makino, M. Sakurai, D.C. Oh, T. Hanada, M.W. Cho, T. Yao, S. Emura, K. Kabayashi, J. Vac. Sci. Technol. B 23, 1308 (2005).

[7] Y.S. Chao, H. Hardtdegen, N. Kaluza, M. von der Ahe, U. Breuer. H.P. Bohem, P. Ruterna, K. Schmalbuch, D. Wenzel, Th. Schapers, B. Beshoten, D. Grutzmacher, H. Luth, J. Cryst. Growth 312, 1 (2009).

[8] S. Krukowski, M. Bockowski, B. Lucznik, I. Grzegory, S. Porowski, T. Suski, Z. Romanowski, J. Phys., Condens. Matter 13, 8881 (2001).

[9] M. Zając, J.B. Gosk, E. Grzanka, M. Kamińska, A. Twardowski, B. Strojek, T. Szyszko, S. Podsiadło, J. Appl. Phys. 93, 4715 (2003).

[10] M. Herbich, W. Mac, A. Twardowski, K. Ando, D. Scalbert, A. Petrou, M. Demianiuk, Phys. Rev. B 58, 1912 (1998).

[11] M. Herbich, W. Mac, A. Twardowski, M. Demianiuk, Phys. Rev. B 59, 2726 (1999). 